\title{
THE RELATION OF GASTRIC FUNCTION TO NAUSEA IN MAN
}

\author{
BY STEWART WOLF 1 \\ (From the 9th General Hospital, U. S. Army, The Neurological Unit, Boston City Hospital, \\ Department of Neurology, Harvard Medical School and the Departments of Medicine \\ and Psychiatry of the New York Hospital and Cornell University \\ Medical College, New York)
}

(Received for publication July 1, 1943)

In this communication are set forth the results of an experimental inquiry into the nature and significance of alterations in gastric function which accompany nausea in man.

Observations were made on the author, on 2 other normal subjects, and on a subject with a large gastric fistula, previously described in connection with other investigations (1). The fistula which had been surgically produced 48 years before because of a benign stricture of the oesophagus, was $3.5 \mathrm{~cm}$. in diameter. The introduction of a lighted anoscope through the stoma afforded a convenient method of examining the gastric mucosa. Moreover, folds of mucosa could be forced out through the stoma and made to lie exposed on the abdominal wall when the subject increased his intra-abdominal pressure. Thus, vascular changes in the mucosa could be quickly recognized and closely followed. These, with other simultaneously recorded gastric functions, were studied for their relationship to the experience of nausea.

\section{METHOD}

The subjects rested supine on a bed, with a thin elastic balloon, inflated to a pressure of $12 \mathrm{~cm}$. of water, in place in the stomach. Continuous tracings of pressure changes in the balloon were recorded on a moving drum. At the same time, continuous aspiration of gastric juice was effected through an ordinary tube. A record of sweating on the forehead was made by taking readings of skin resistance every minute by means of an ordinary Wheatstone bridge resistance-measuring device, ${ }^{2}$ and finally, observations were made of changes in the subject's pulse rate and in the color of his face.

In the fistulous subject, in addition to these determinations, vascular changes in the mucosa were observed by comparing color changes to a standard color scale. It has been shown in another publication that these changes

\footnotetext{
1 Captain M. C., A. U. S.

2 In making the measurements of skin resistance, the help of Dr. Charles Berry of the Department of Anatomy, Cornell University Medical School, is gratefully acknowledged.
}

in color actually parallel changes in blood flow (2). Salivary secretion was also estimated by emptying the blind upper end of the oesophagus before observations were begun, and measuring the accumulation of saliva at appropriate intervals.

\section{OBSERVATIONS}

Ten experiments were carried out after the manner described, in addition to 15 others in which only measurements of gastric motor activity were recorded. Nausea was induced by caloric vestibular stimulation, swinging, rotation of the head, and by situations involving a reaction of fear and withdrawal. The stimuli were applied at intervals, from 1 to 15 hours after feeding. After a suitable control period, 1 external auditory canal was irrigated with cold water. Note was made of the onset of nystagmus, as well as of the occurrence of vertigo, nausea, or other sensations. The degree of discomfort experienced by the subjects during irrigation varied roughly with the temperature of the water and the duration of the stimulus. The longer the period of irrigation and the colder the water, the greater was the intensity of discomfort. The following protocols illustrate the gastric and other bodily changes which occurred during vestibular stimulation.

Protocol 1. Near the beginning of a phase of spontaneously accelerated gastric activity, the left canal was irrigated with water at $5^{\circ} \mathrm{C}$. Within 1 minute, nystagmus and vertigo appeared. At this point, the gastric contractions decreased in amplitude. No other changes were noted as yet, however. Skin resistance on the forehead remained unchanged until 5 minutes later when the first vague feelings of nausea appeared. Coincident with this, a sharp fall in skin resistance from $1,000,000$ ohms occurred, reaching 90,000 ohms within 1 minute. A minute after that, perspiration appeared clearly on the forehead. Moderate tachycardia and facial pallor accompanied these changes. No gastric contractions occurred during the period of nausea. There was frequent coughing but no actual vomiting (Figure 1). Unfortunately, in this experiment, no record of duodenal contractions was obtained. Ingelfinger (3) has shown, however, that the 


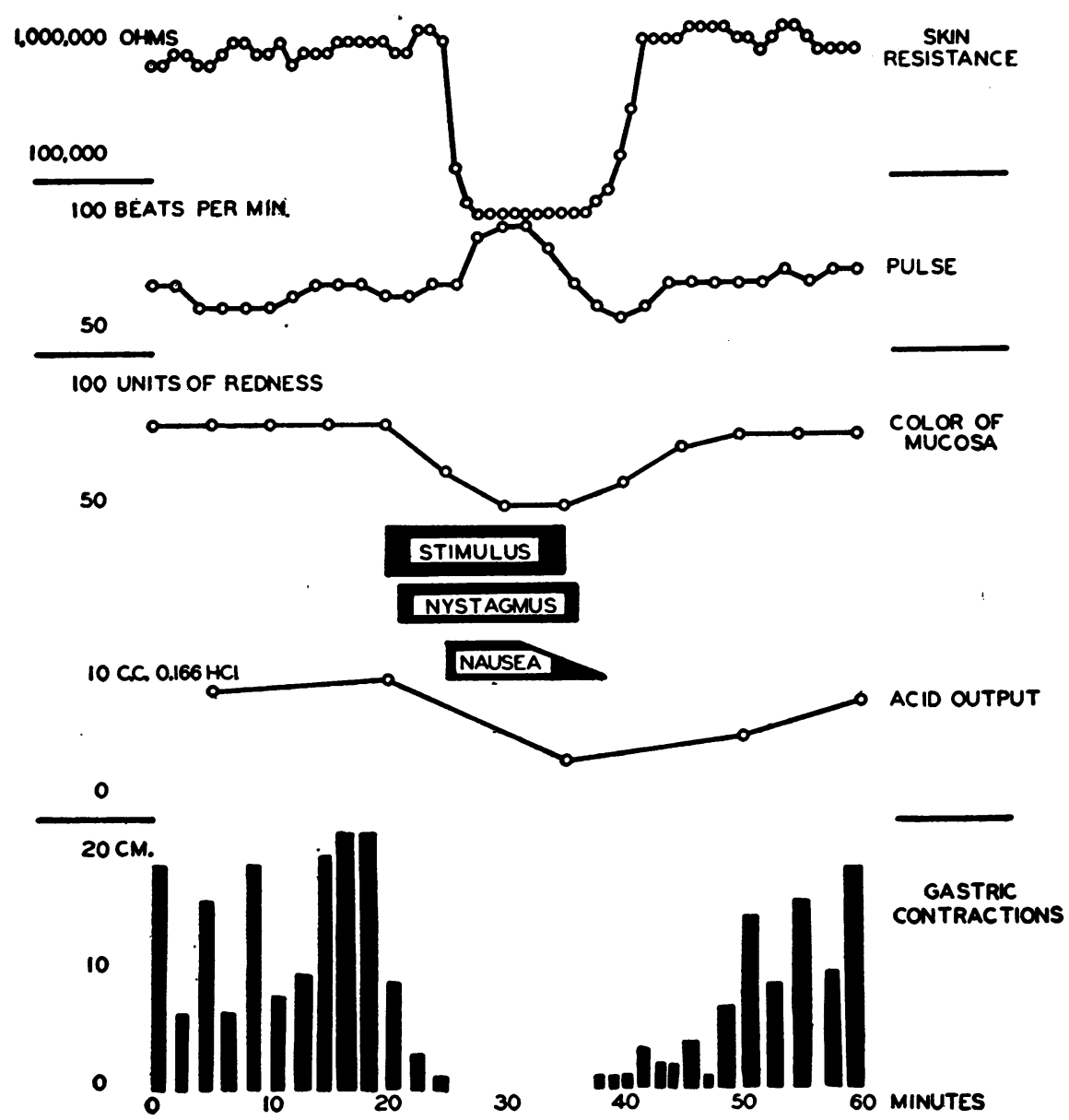

Fig. 1. Gastric and Other Bodily Changes, Associated with Nausea, Induced by Caloric Vestibular Stimulation

contractile state of the duodenum increases as that of the stomach decreases, so that eventually, by the difference in pressure gradient, the duodenal balloon is forced back into the stomach.

Comment. The striking finding in this experiment was the early inhibition of gastric motor activity and the much later onset of the other bodily changes. It would be of interest to know how many of these latter would follow such a stimulus if actual nausea did not occur. The opportunity to make this differentiation became available in other experiments in which lesser degrees of discomfort occurred.

Protocol 2. Using water at $8^{\circ} \mathrm{C}$., 3 minutes after vestibular stimulation was undertaken, there occurred slight vertigo and nystagmus, as well as a distinctly uncomfortable "sinking" feeling without actual nausea. Concomitant with this feeling of discomfort, there occurred a sharp drop in skin resistance on the forehead from $1,900,000$ ohms to 100,000 ohms within a minute and a half. With the disappearance of the uncomfortable sensations, the skin resistance quickly rose to its former level. Apart from the usual reduction in the amplitude of gastric contractions which occurred within 1 minute, no measurable changes occurred in the stomach and there were no changes in the output of saliva.

Comment. In this experiment, reduction in the amplitude of gastric contractions antedated the onset of nystagmus and it was the only change which was noted in the stomach. Lowering of skin resistance on the forehead came on later and was transitory, corresponding in point of time to a feeling of slight abdominal discomfort.

Protocol 3. When slightly warmer water at $12^{\circ} \mathrm{C}$. was used, the onset of nystagmus was delayed until 5 minutes after the start of vestibular stimulation. It was not marked and vertigo was not troublesome. No nausea occurred. In the stomach, contractions which were of 
considerable amplitude when stimulation was started decreased markedly 1 minute after the stimulus was applied. The mucous membrane of the stomach became slightly less red but no change in the rate of acid secretion, output of gastric mucus, or saliva could be detected. Neither did there occur any change in the appearance of the face or in the pulse rate. In this experiment, and in others in which the subject recognized little or no discomfort, there occurred no significant change in skin resistance on the forehead. The findings are shown graphically in Figure 2.

Comment. This experiment has shown that when the stimulus failed to induce significant discomfort, the only bodily change observed was a sharp decrease in the vigor of gastric contractions.

Protocol 4. In another subject, 2 minutes after the commencement of irrigation, the same sort of discomfort described in the previous protocol occurred. It was associated with vigorous nystagmus and vertigo. Skin resistance fell suddenly from 180,000 ohms to $90,000 \mathrm{ohms}$. The palms became moist and the pulse rate rose from 65 to 90 beats per minute. A minute later, when the fleeting distress had passed away, the skin resistance and pulse rate returned to their former level. Nystagmus and vertigo, however, continued.

Protocol 5. In a third subject, the effects described above were evident 2 minutes after the start of vestibular stimulation. The skin resistance on the forehead fell from 650,000 ohms to 210,000 ohms and 2 minutes later had returned to $670,000 \mathrm{ohms}$, despite the persistence of nystagmus and vertigo.

Comment. These observations indicate that peripheral autonomic manifestations were not necessarily an accompaniment of vertigo but rather were associated with the feeling of abdominal discomfort and mild apprehension. As shown in the first protocol, with the greater discomfort of actual nausea, these autonomic effects were even more marked.

Swing sickness. A swing, consisting of a chair suspended by cables from a bar 18 feet above the
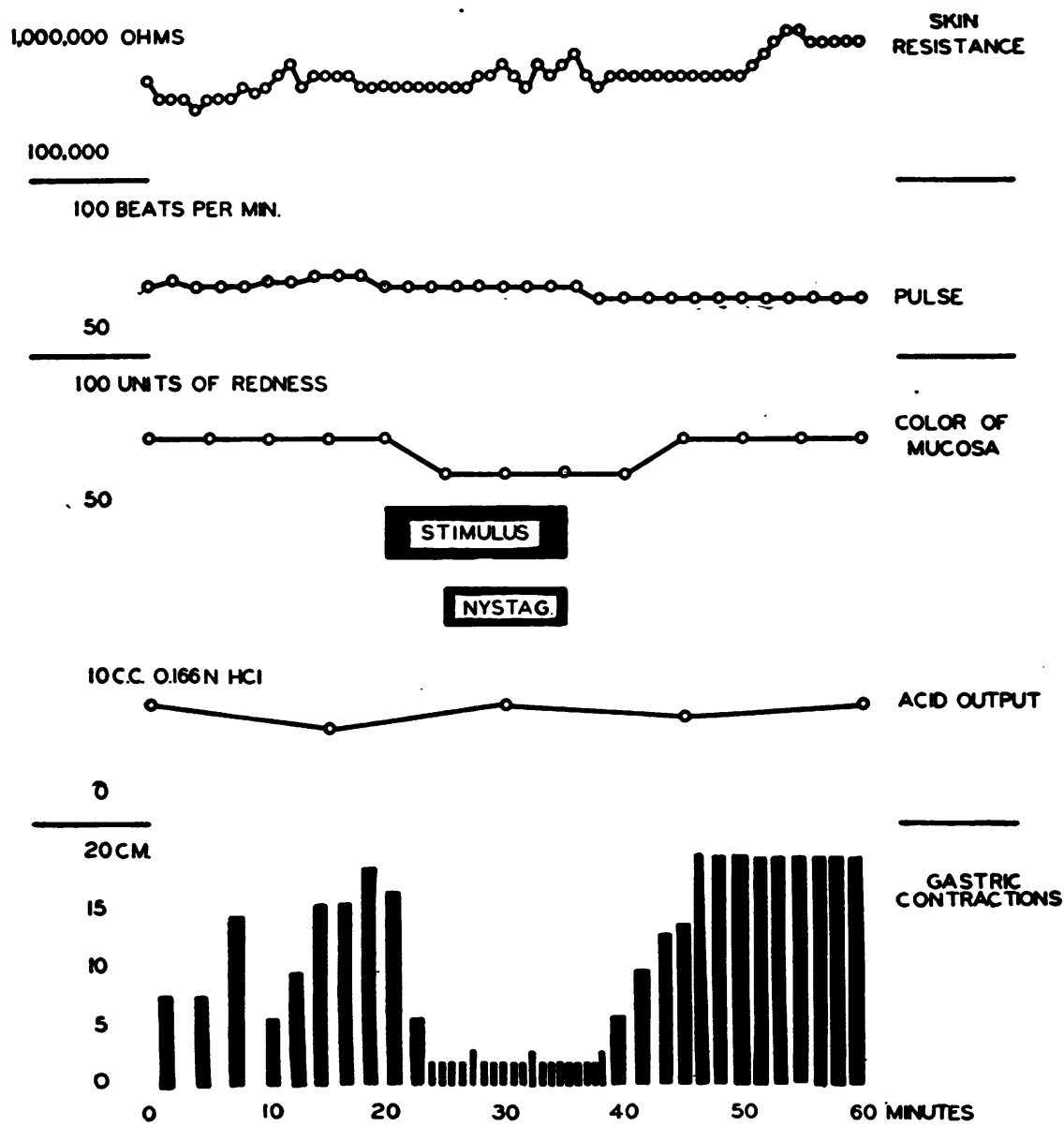

Fig. 2. Gastric and Other Bodily Changes following Caloric Vestibular Stimulation Which Failed to Induce Nausea 
seat, was used. The subject was placed in the chair and swung through an arc of $90^{\circ}$, at the rate of approximately 15 complete swings a minute.

Protocol 6. Swinging was undertaken during a phase of vigorous gastric contractions. After 3 minutes, the contractions decreased sharply in amplitude and started up again 5 minutes after the swinging was stopped. There occurred no nystagmus, vertigo, nausea, or other discomforts of any sort. No change in the rate of acid or mucus secretion was noted. Salivary flow was not altered and no pallor, tachycardia, or sweating occurred.

Protocol 7. An earlier swing experiment was attended with considerably more discomfort than the one described in the previous protocol. Swinging was carried out under similar circumstances but after 20 minutes of swinging, the subject felt "weak in the knees," "light-headed," and slightly nauseated. The stomach had become pale. The vigorous contractions had disappeared and acid production had decreased. The results are shown in Figure 3.
Comment. These experiments show that the same bodily changes were induced by swinging as by caloric vestibular stimulation. Decreased gastric motility was a constant occurrence but further gastric changes and the more widespread autonomic effects occurred only when nausea or discomfort was induced.

Nausea accompanying fear. Several opportunities have been available to observe the changes in the function of our fistulous subject's stomach during alarm fear, and a reaction of withdrawal. They have been commented upon at length in another publication (4) but may be reviewed briefly here.

During a period of relatively accelerated gastric functioning, while the mucosa was under observation and the gastric juice was being collected, a

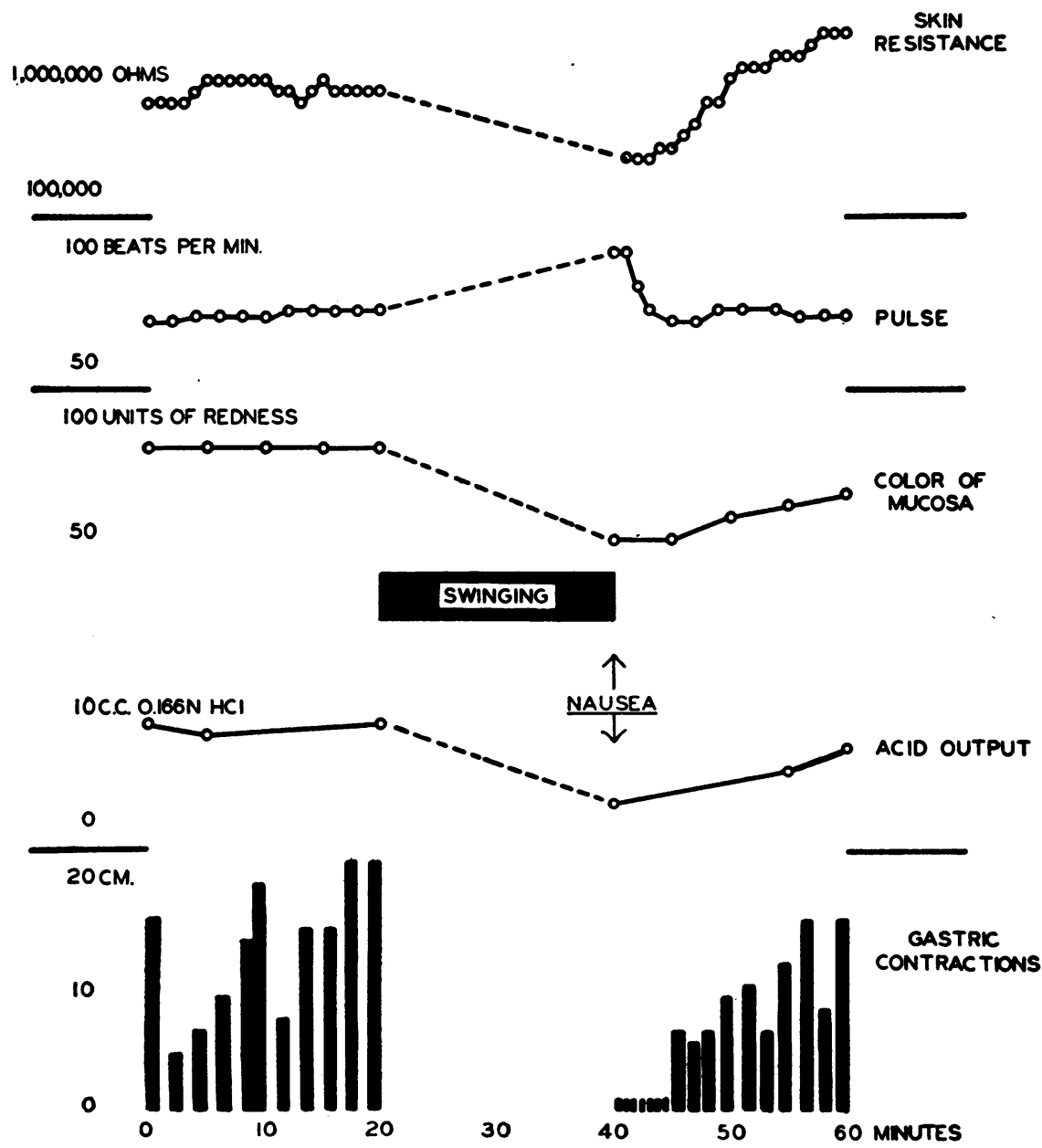

Fig. 3. Gastric and Other Bodily Changes Associated with Nausea INDUCED BY SWINGING 


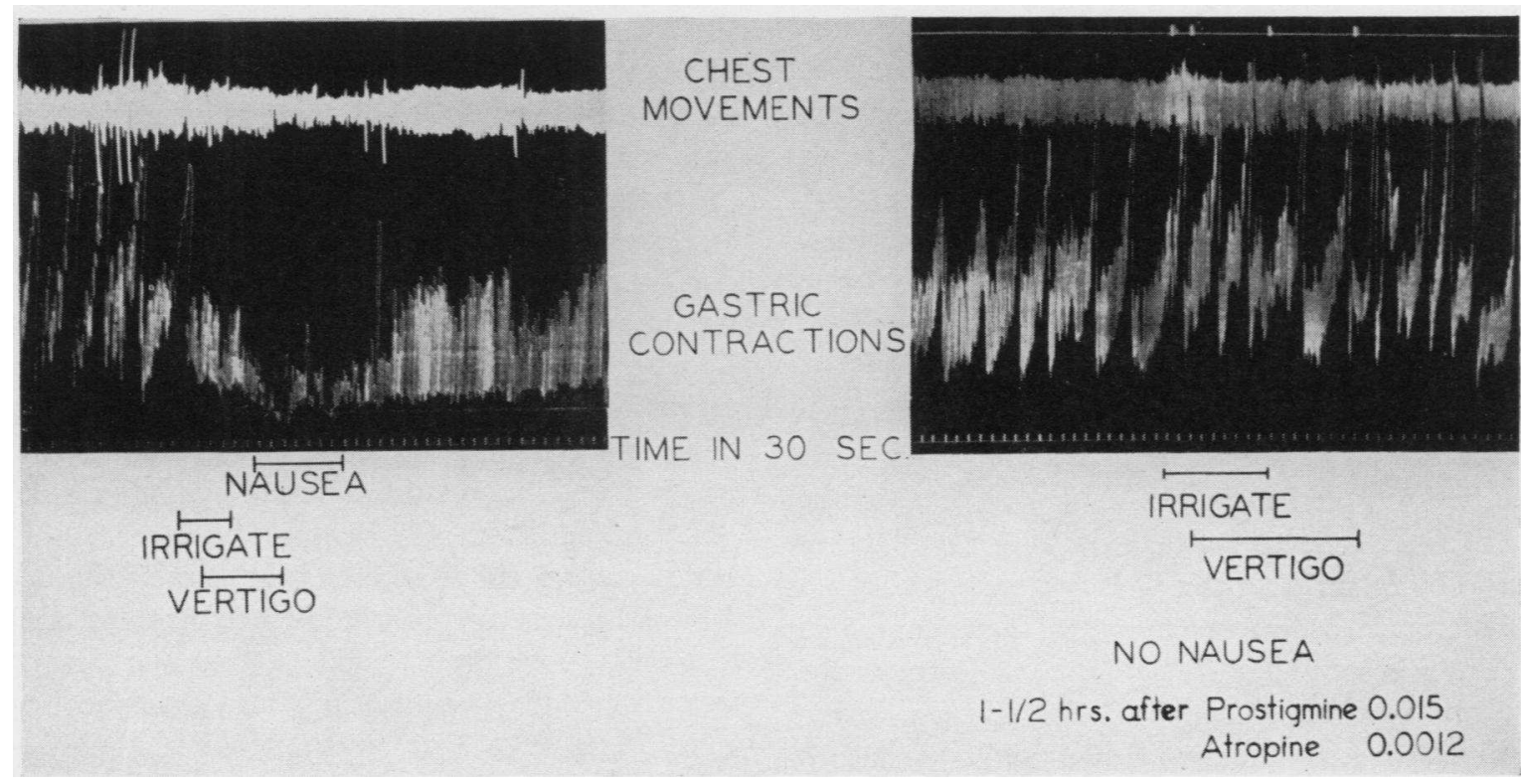

Fig. 4. Kymographic Tracings of Gastric Contractions

On the left is illustrated the usual sudden cessation of gastric contractions, decrease in gastric tone, and the occurrence of nausea, following irrigation of the auditory canal with cold water.

The tracing on the right was made from the same subject, one and one-half hours after prostigmine and atropine. Despite irrigation with water of the same temperature as the control $\left(2^{\circ} \mathrm{C}\right.$. $)$ for a period twice as long, no interruption of gastric motor activity occurred, and no nausea was experienced.

member of the staff entered the room and began searching impatiently for certain protocols to which he attached particular importance. As he searched, he muttered imprecations. Our subject, who tidied up the laboratory the previous night, had mislaid them. He was fearful of detection and of losing his job. He lay motionless on the table. His face became pale, as did his gastric mucosa, and acid output decreased temporarily until the doctor located his papers. On numerous other occasions, it was noted that the subject suffered depression of gastric function associated with pallor and sweating in situations involving fear and a feeling of withdrawal. Frequently nausea occurred in such a setting.

Nausea following other stimuli. Nausea was induced in the subjects by 2 other methods, namely, by rotating the head rhythmically while the eyes fixed some object on the ceiling and by administering a mixture of $5 \mathrm{~cm}$. of powdered mustard in $100 \mathrm{cc}$. of water. In each case, the changes in gastric behavior already described occurred, the earliest and most striking alteration being the prompt cessation of gastric contractions, with a decrease in tone of the stomach wall.

\section{The relation of gastric motor activity to the sensation of nausea}

It is clear that the changes in motor activity which occurred in these experiments did not result from nausea since they antedated the onset of the sensation and occurred with stimuli of insufficient intensity to cause nausea. These data do not determine, however, whether or not the altered gastric motility is essential to the occurrence of nausea. In order to settle this question, an attempt was made to prevent by drugs the inhibition of gastric contractions which followed nauseating stimuli. Veach (5) and his associates found that a combination of prostigmine and atropine, administered hypodermically, uniformly induced vigorous contractile activity in the atonic stomach of man. In the following experiments, a combination of these drugs given by mouth was tried out as a prophylactic against nausea.

Action of a combination of prostigmine and atropine on the gastric motor mechanism. In 6 experiments on 3 subjects, prostigmine hydrobromide, 0.015 gram, and atropine sulphate, 0.0012 gram, were administered while recordings of gas- 
tric contractions were being made. In each case, vigorous contractile activity began within 10 to 30 minutes, if it was not already in progress, and the contractions continued for more than 2 hours. At intervals of half an hour and one and one-half hours, irrigation of the auditory canal was carried out for 5 minutes with water at $2^{\circ} \mathrm{C}$. In no case was there any interruption of the motor activity and, despite vigorous nystagmus and vertigo, no nausea occurred. Similarly rhythmic rotation of the subject's head while the eyes fixed a spot on the ceiling failed to induce nausea or to alter the pattern of gastric motility. Gagging was then induced by stimulating the posterior pharynx with a tongue blade. Still gastric contractions continued and no nausea was felt. Figure 4 illustrates the contrast in motor function of the stomach during vestibular stimulation, with and without the administration of prostigmine and atropine. No detectable side reactions attended the administration of this combination of drugs.

Effects of prostigmine and atropine alone. When either of these drugs was administered alone in the dosage stated, no predictable effect on gastric motility was induced. Prostigmine was usually followed by the appearance of vigorous gastric contractions, but often the intestinal cramps which also occurred resulted in nausea and, with the nausea, there occurred inhibition of gastric contractions. The administration of atropine alone was also associated with undesirable side effects, such as dryness of the mouth (1). No detectable side reactions attended the administration of both drugs in the combination noted above.

Comment. The fact that nausea may be prevented, despite strong nauseating stimuli, by controlling with drugs the pattern of gastric motility indicates that gastric relaxation and hypomotility are essential to the occurrence of nausea. They do not, however, allow any conclusions regarding the mechanism of nausea or the mode of mediation of that sensation.

\section{SU M MARY}

Changes in gastric function during nausea have been explored and correlated with other more widespread changes in bodily function. Several nauseating stimuli were applied. The changes as- sociated with the nausea resulting from each were clear-cut and uniform.

Following stimuli of intensity insufficient to cause nausea, there occurred interruption of gastric contractions and decreased muscular tone of the stomach wall. Stronger stimuli, which caused actual nausea, induced further changes in the stomach, characterized by pallor, decrease in the output of hydrochloric acid, and acceleration of the production of mucus. More widespread bodily changes were also noted, including salivation, sweating, and tachycardia followed by bradycardia.

Nausea occurred only during phases of inhibition of gastric motor activity. When, by the use of a combination of prostigmine and atropine, gastric contractions were made to continue despite strong vestibular stimulation, nausea failed to occur. The fact that gastric motor inhibition could be blocked by drugs, thereby preventing nausea, suggests their possible prophylactic use against seasickness.

\section{CONCLUSIONS}

1. Nausea induced by several stimuli was found to be accompanied by definite and uniform changes in gastric function.

2. While it cannot be said what connection these changes have with its mechanism, nausea occurred only during gastric relaxation and hypomotility.

3. By controlling pharmacologically the gastric motor mechanism before the application of stimuli, it was possible to prevent the occurrence of nausea.

\section{BIBLIOGRAPHY}

1. Wolf, S., and Wolff, H. G., Human Gastric Function, An Experimental Study of a Man and His Stomach. Oxford University Press, New York City, 1943.

2. Richards, C. H., Wolf, S., and Wolff, H. G., The measurement and recording of gastroduodenal blood flow in man by means of a thermal gradientometer. J. Clin. Invest., 1942, 21, 551.

3. Ingelfinger, F. J., Behavior of the duodenum during nausea. Bull. New England M. Center, 1942, 4, 120.

4. Wolff, H. G., and Wolf, S., Studies on a subject with a large gastric fistula. Changes in the function of the stomach in association with varying emotional states. Tr. A. Am. Physicians, 1942, 57, 115.

5. Veach, H. O., Lauer, B. R., and James, A. G., Effects of prostigmin and atropine on the human stomach. J. Pharmacol. and Exper. Therap., 1938, 62, 422. 